\title{
Convergence of Global Solutions to the Cauchy Problem for the Replicator Equation in Spatial Economics
}

\author{
Minoru Tabata ${ }^{1}$ and Nobuoki Eshima ${ }^{2}$ \\ ${ }^{1}$ Department of Mathematical Sciences, Osaka Prefecture University, Sakai, Osaka 599-8531, Japan \\ ${ }^{2}$ Department of Statistics, Oita University, Oita 879-5593, Japan \\ Correspondence should be addressed to Minoru Tabata; mnrtabata@luck.ocn.ne.jp
}

Received 21 March 2016; Accepted 29 June 2016

Academic Editor: Douglas R. Anderson

Copyright @ 2016 M. Tabata and N. Eshima. This is an open access article distributed under the Creative Commons Attribution License, which permits unrestricted use, distribution, and reproduction in any medium, provided the original work is properly cited.

We study the initial-value problem for the replicator equation of the $N$-region Core-Periphery model in spatial economics. The main result shows that if workers are sufficiently agglomerated in a region at the initial time, then the initial-value problem has a unique global solution that converges to the equilibrium solution expressed by full agglomeration in that region.

\section{Introduction}

The applications of functional equation theory (FET) have been broadened from natural sciences to various disciplines of social sciences. In turn, it accelerates the progress of FET to mathematically study various functional equations considered in social sciences (see, e.g., [1]). If we attempt to study such new applications of FET in social sciences, then we should concentrate on spatial economics. Spatial economics is an interdisciplinary area between economics and geography. Its purpose is to study the location, distribution, and selforganization of economic activities. In about 1990, Krugman began important new research in this interdisciplinary area, and his research has since grown into a major branch of economics known as the New Economic Geography (NEG). In 2008, the Nobel Prize in Economic Sciences was awarded to Krugman for his remarkable contributions. In the NEG, many interesting nonlinear functional equations have been considered. Hence the NEG is one of the most promising fields of application for FET. However, little mathematical research has been conducted on these nonlinear functional equations (see [2-12]).

One of the most important models in the NEG is the Nregion Core-Periphery (NCP) model (see [2-13]). The economy in this model consists of agriculture and manufacturing. Agriculture and manufacturing are conducted in a finite set of points that represent economic regions. In this model an equilibrium is studied fully in $[2,3,14,15]$. However, it is more important to consider dynamic models (see, e.g., [1618]). Hence, Krugman constructs an evolutionary game by combining the NCP model with replicator dynamics in [4, p. 77]. This evolutionary game is referred to as the NCP evolutionary game (NCPEG), and the replicator equation thus combined is referred to as the NCPEG replicator equation.

This paper deals with the Cauchy problem for the NCPEG replicator equation. This Cauchy problem is studied numerically when the time variable is sufficiently small [4, Chapter 6]. However, there are no full mathematical studies on the behavior of global solutions as the time variable tends to infinity. We can derive a simple nonlinear equation from the NCP model in an urban setting, that is, under the condition that the economy has no agriculture [15]. By making use of this simplification, we have studied the NCPEG replicator equation [19]. However, the condition of an urban setting is too restrictive. Hence, we should study the NCPEG in an urban-rural setting, that is, under the general condition that the economy consists of both manufacturing and agriculture. The method in [19] cannot be applied to the NCPEG in an urban-rural setting, since it essentially depends on the simplification attained by accepting the condition of an urban setting. In what follows, we simply refer to the NCP model in an urban-rural setting, the NCPEG in an urban-rural setting, and the NCPEG replicator equation in an urban-rural setting 
as the NCP model, the NCPEG, and the NCPEG replicator equation, respectively. No confusion should arise.

The pure-strategy set of NCPEG is defined as a finite set of points (economic regions) at which manufacturing and agriculture are conducted. The distribution of workers is defined as a solution to the Cauchy problem for the NCPEG replicator equation. The coefficient of the NCPEG replicator equation denotes that the growth rate of the worker population choosing a strategy (i.e., an economic region) is equal to the difference between the average payoff and the payoff in the chosen region. Payoffs are defined as the distribution of real wages. Hence, workers move toward regions that offer higher real wages and away from regions that offer below-average real wages $[4$, p. 62$]$.

If we regard the distribution of workers as a given function in the nominal wage equation of the NCP model, then the equation has a unique solution [14]. By calculating the distribution of real wages from this unique solution, we can construct an operator that maps the distribution of workers, which is contained as an unknown function in the NCPEG replicator equation, to the distribution of real wages (payoffs). Therefore, we can regard the NCPEG replicator equation as a nonlinear ordinary differential equation whose coefficient is expressed by the nonlinear operator acting on an unknown function of the equation. Hence, the NCPEG replicator equation is quite a new kind of nonlinear ordinary differential equation, in contrast to the coefficients of the usual replicator equations, which are expressed by explicit functions of an unknown function (see, e.g., [20]).

In this study we prove that if the transport costs are sufficiently small, then the Cauchy problem for the NCPEG replicator equation has a unique global solution (Theorem 1). Furthermore, we prove that if the black-hole condition and the triangular inequality for transport costs hold along with the sufficient conditions imposed on Theorem 1 and if workers are sufficiently agglomerated in a region at the initial time, then the initial-value problem has a unique global solution that converges to full agglomeration in that region as the time variable tends to infinity (Theorem 3 ).

\section{Fundamental Equations}

By $D$ we denote the pure-strategy set of the NCPEG, where $D$ is a finite set of points contained in an Euclidean space. Each point of $D$ represents a pure strategy (region). No restriction is imposed on the total number of regions. For mathematical generality, we impose no restriction on the dimension of the Euclidean space. By $L(D)$ we denote the set of all real-valued functions of $x \in D$. By $L_{+}(D)$ we denote the set of all positivevalued functions of $x \in D$. We define the following norms in $L(D)$ :

$$
\begin{aligned}
\|f\| & :=\max _{y \in D}|f(y)|, \\
\|f\| \mid & :=\sum_{y \in D}|f(y)| .
\end{aligned}
$$

By $\Delta$ we denote the set of all nonnegative-valued functions $f=f(x) \in L(D)$ such that $|\|f\||=1$. By $C_{0+}^{1}([0,+\infty))$ we denote the set of all nonnegative-valued functions of $(t, x) \in$ $[0,+\infty) \times D$ that are continuous with respect to $t \geq 0$ and continuously differentiable with respect to $t>0$ for each $x \in$ D.

The NCP model is described by the nominal wage equation, which is the following nonlinear discrete equation (see $[4,(5.5)])$ :

$$
\begin{aligned}
& w(x)^{\sigma}=\sum_{y \in D} Y(\lambda(y), w(y)) G_{\sigma}(\lambda(\cdot), w(\cdot) ; y)^{\sigma-1} \\
& \cdot \exp (-(\sigma-1) c(x, y))
\end{aligned}
$$

where $w=w(x)$ is an unknown function that denotes the distribution of nominal wages. We denote the distribution of workers by $\lambda=\lambda(x)$ and the elasticity of substitution by $\sigma$. Income $Y=Y(\lambda(y), w(y))$ has the following form (see [4, (5.3)]):

$$
Y(\lambda(y), w(y))=\mu \lambda(y) w(y)+(1-\mu) \phi(y),
$$

where we denote the share of manufacturing expenditure by $\mu$ and the distribution of farmers by $\phi=\phi(y)$. The price index $G_{\sigma}=G_{\sigma}(\lambda(\cdot), w(\cdot) ; x)$ is the following nonlinear operator that acts on $\lambda=\lambda(x)$ and $w=w(x)$ (see $[4,(5.4)]$ ):

$$
\begin{aligned}
& G_{\sigma}(\lambda(\cdot), w(\cdot) ; x)^{\sigma-1} \\
& \quad:=\frac{1}{\sum_{y \in D} \lambda(y)(1 / w(y))^{\sigma-1} \exp (-(\sigma-1) c(x, y))} .
\end{aligned}
$$

We reasonably accept the following condition in the NEG.

Condition 1.

$$
\begin{aligned}
\sigma & >1, \\
0 & <\mu<1, \\
\phi & =\phi(x) \in \Delta, \\
c(x, x) & =0 \quad \text { for each } x \in D, \\
c(x, y) & =c(y, x) \quad \text { for each } x, y \in D, \\
c(x, y) & >0 \quad \text { if } x \neq y .
\end{aligned}
$$

In [14, (3.8), Lemma 3.2] we define a positive-valued function $V=V(\mu, \sigma)$ such that

$$
\begin{aligned}
& 1<V(\mu, \sigma)<\frac{1}{\mu} \\
& \qquad \text { for each }(\mu, \sigma) \in(0,1) \times(1,+\infty) .
\end{aligned}
$$

By making use of this function, we assume that the transport costs are sufficiently small compared with the elasticity of substitution $\sigma$ and the share of manufacturing expenditure $\mu$, as follows.

Condition 2.

$$
\mathbf{C}<\left(\frac{1}{(\sigma-1)}\right) \log V(\mu, \sigma),
$$

where $\mathbf{C}:=\max _{x, y \in D} c(x, y)$. 
It is proved that if Conditions 1 and 2 hold, then (2) has a unique solution $w=w(x) \in L_{+}(D)$ [14, Theorem 3.3]. Making use of this result, we can define an operator that maps the distribution of workers,

$$
\lambda=\lambda(x) \in \Delta,
$$

to the distribution of nominal wages $w=w(x) \in L_{+}(D)$. We denote this operator by

$$
P_{1}=P_{1}(\lambda(\cdot) ; x)
$$

The distribution of real wages $\omega=\omega(x)$ is defined as follows (see $[4,(5.6)])$ :

$$
\omega=\omega(x):=\frac{w(x)}{G_{\sigma}(\lambda(\cdot), w(\cdot) ; x)^{\mu}} .
$$

Replacing $w=w(x)$ by $P_{1}=P_{1}(\lambda(\cdot) ; x)$ in (15), we can define an operator that maps the distribution of workers (13) to the distribution of real wages $\omega=\omega(x)$ as follows:

$$
P_{2}(\lambda(\cdot) ; x)=\frac{P_{1}(\lambda(\cdot) ; x)}{G_{\sigma}\left(\lambda(\cdot), P_{1}(\lambda(\cdot) ; \cdot) ; x\right)^{\mu}} .
$$

We define the average real wage as follows (see $[4,(5.1)]$ ):

$$
m(\lambda(t, \cdot), \omega(t, \cdot)):=\sum_{y \in D} \lambda(t, y) \omega(t, y)
$$

where $\lambda=\lambda(t, x)$ denotes the distribution of workers at time $t \geq 0$ and

$$
\omega(t, x):=P_{2}(\lambda(t, \cdot) ; x) .
$$

The NCPEG replicator equation has the following form (see $[4,(5.2)])$ :

$$
\left(\frac{d}{d t}\right) \lambda(t, x)=\mathbf{M}(\lambda(t, \cdot) ; x) \lambda(t, x),
$$

where

$$
\begin{aligned}
& M(\lambda(t, \cdot), \omega(t, \cdot) ; x):=\omega(t, x)-m(\lambda(t, \cdot), \omega(t, \cdot)), \\
& \mathbf{M}(\lambda(t, \cdot) ; x):=M\left(\lambda(t, \cdot), P_{2}(\lambda(t, \cdot) ; \cdot) ; x\right) .
\end{aligned}
$$

This equation is quite a new kind of nonlinear ordinary differential equation as mentioned in the Introduction. We consider the Cauchy problem for (19) with the initial condition,

$$
\lambda(0, x)=\lambda_{0}(x) \in \Delta
$$

where $\lambda_{0}=\lambda_{0}(x)$ is a given function.

\section{Global Solutions}

From $[14,(5.11)],(5)$, and (6), we easily obtain the following inequality:

$$
0<m_{1}<1<m_{2}
$$

where

$$
\begin{aligned}
m_{1} & :=\frac{(\exp (-\mu \mathbf{C})) R_{1}^{1 / \sigma}}{R_{2}^{\mu / \sigma}} \\
m_{2} & :=\frac{R_{2}^{1 / \sigma}}{R_{1}^{\mu / \sigma}} \\
R_{i} & :=\left\{\frac{(1-\mu)}{\left(\exp \left((-1)^{i+1} \alpha\right)-\mu\right)}\right\}^{\sigma}, \quad i=1,2, \\
\alpha & =\alpha(\sigma, \mathbf{C}):=(\sigma-1) \mathbf{C} .
\end{aligned}
$$

Theorem 1. If Conditions 1 and 2 hold, then the following statements (i) and (ii) hold:

(i) The Cauchy problem for (19) with (22) has a unique global solution $\lambda=\lambda(t, x) \in C_{0+}^{1}([0,+\infty))$.

(ii) The global solution $\lambda=\lambda(t, x)$ satisfies the following statements:

$$
\begin{gathered}
\lambda=\lambda(t, x) \in \Delta \quad \text { for each } t \geq 0, \\
\lambda_{0}(x) e^{-\Delta m t} \leq \lambda(t, x) \leq \lambda_{0}(x) e^{\Delta m t} \\
\quad \text { for each }(t, x) \in[0,+\infty) \times D, \\
\lambda(t, x) \leq 1-\left(1-\lambda_{0}(x)\right) e^{-\Delta m t} \\
\quad \text { for each }(t, x) \in[0,+\infty) \times D, \\
\left\|\left(\frac{d}{d t}\right) \lambda(t, \cdot)\right\| \leq \Delta m \quad \text { for each } t>0, \\
m_{1} \leq \omega(t, x) \leq m_{2} \\
\quad \text { for each }(t, x) \in[0,+\infty) \times D, \\
\|\omega(r, \cdot)-\omega(s, \cdot)\| \leq \beta \Delta m|r-s| \quad \text { for each } r, s \geq 0,
\end{gathered}
$$

where

$$
\Delta m:=m_{2}-m_{1},
$$

$\omega=\omega(t, x)$ is defined by (18), and $\beta$ is a constant dependent on $(\mu, \sigma, \mathbf{C})$ such that

$$
\begin{aligned}
& \beta>1 \\
& \quad \text { for each }(\mu, \sigma, \mathbf{C}) \in(0,1) \times(1,+\infty) \times(0,+\infty) .
\end{aligned}
$$

This theorem is proved in the last section, where the constant $\beta$ is defined explicitly as a function of $(\mu, \sigma, \mathbf{C}) \epsilon$ $(0,1) \times(1,+\infty) \times(0,+\infty)$. We also use the constant $\beta$ in Theorem 3. We must note that Conditions 1 and 2 are the same as those accepted in [14] to ensure the existence and uniqueness of a short-run equilibrium in the NCP model. Making use of (26) and (27), we obtain the following corollary.

Corollary 2. Assume that Conditions 1 and 2 hold. If $0<$ $\lambda_{0}(x)<1$ for some $x \in D$, then $0<\lambda(t, x)<1$ for each $t \geq 0$. 
From this corollary we see that if workers exist in a region at $t=0$, then there are workers in the region for each $t>0$.

\section{Convergence of Global Solutions}

We require the following condition to prove Theorem 3.

\section{Condition 3.}

$$
\begin{aligned}
& c(z, y) \leq c(z, x)+c(x, y) \text { for each } x, y, z \in D, \\
& \frac{(\sigma-1)}{\sigma} \leq \mu .
\end{aligned}
$$

Inequality (33) is the triangle inequality for transport costs. We accept this inequality, since transport costs generally and reasonably increase with distance. If $c(x, y)=$ $\tau|x-y|$, where $\tau$ is a positive constant, then (8), (9), (10), and (33) hold. Inequality (34) corresponds to the black-hole condition, which complements the assumption of no black holes, $[4,(4.45)]$. Condition (34) plays a role in making the forces working toward agglomeration prevail [4, p. 58].

Theorem 3. Assume that Conditions 1, 2, and 3 hold. If there exists $X \in D$ such that

$$
0<1-\lambda_{0}(X)<\delta
$$

where

$$
\begin{aligned}
& \delta:=\frac{(1 / 4) \gamma}{\beta}, \\
& \gamma:=1-\{1-\mu(1-\exp (-2(\sigma-1) \mathbf{c}))\}^{1 / \sigma}, \\
& \mathbf{c}:=\min _{x, y \in D, x \neq y} c(x, y),
\end{aligned}
$$

and $\beta$ is the positive constant employed in Theorem 1, then the following inequalities hold for each $t \geq 0$ :

$$
\begin{aligned}
\Lambda(t) & \leq \lambda(t, X)<1, \\
0 & \leq \lambda(t, x) \leq 1-\Lambda(t)
\end{aligned}
$$

for each $x \neq X$,

\|\|$\lambda(t, \cdot)-\lambda_{\infty}(X, \cdot) \| \mid \leq 2(1-\Lambda(t))$,

$$
\begin{aligned}
\left(\frac{d}{d t}\right) \lambda(t, X) & \geq F(\lambda(t, X)), \\
\left\|\omega(t, \cdot)-\omega_{\infty}(X, \cdot)\right\| & \leq 2 \beta(1-\Lambda(t)), \\
\omega(t, X)-\omega(t, x) & \geq \rho \quad \text { for each } x \neq X,
\end{aligned}
$$

where $\omega=\omega(t, x)$ is defined by (18) and

$$
\begin{aligned}
& \Lambda=\Lambda(t):=\frac{\lambda_{0}(X)}{\left\{\lambda_{0}(X)+\left(1-\lambda_{0}(X)\right) \exp (-\rho t)\right\}} \\
& \rho:=(1-\kappa) \gamma \\
& \kappa:=\frac{\left(1-\lambda_{0}(X)\right)}{\delta}, \\
& F(z):=\rho(1-z) z, \\
& \lambda_{\infty}(X, x):=1 \quad \text { if } x=X ; \\
& \lambda_{\infty}(X, x):=0 \quad \text { for each } x \neq X, \\
& \omega_{\infty}(X, x):=e^{-\mu c(X, x)} w_{\infty}(X, x), \\
& w_{\infty}(X, x):=\left\{\mu e^{-(\sigma-1) c(X, x)}\right. \\
& \left.+(1-\mu) \sum_{y \in D} \phi(y) e^{(\sigma-1)(c(X, y)-c(x, y))}\right\}
\end{aligned}
$$

This theorem is proved in the last section. Each $\lambda(x) \in \Delta$ satisfies that

$$
\lambda(x)=1-\sum_{y \in D, y \neq x} \lambda(y) \quad \text { for each } x \in D .
$$

It follows from (35) and (52) that

$$
\begin{aligned}
1-\delta & <\lambda_{0}(X)<1, \\
0 & <\sum_{y \in D, y \neq X} \lambda_{0}(y)<\delta .
\end{aligned}
$$

Making use of (5), (6), and (10), we obtain

$$
0<\gamma<1
$$

Hence, recalling (32), we deduce that

$$
0<\delta<\frac{1}{4}
$$

Applying (55) to (53), we can say that workers are sufficiently agglomerated in region $X \in D$ at $t=0$ and that the total number of workers in other regions is sufficiently small at $t=$ 0 .

Applying (35) and (54) to (46), we obtain

$$
0<\rho<1 \text {. }
$$

Applying (56) to (45), we deduce that $\Lambda(t)$ is a monotoneincreasing function of $t \geq 0$ and that

$$
\Lambda(t) \longrightarrow 1-0 \quad \text { as } t \longrightarrow+\infty .
$$

Substitute (49) in (2) and (4). Making use of Condition 1 and noting that $w(X)=1$, we can solve the equations thereby 
obtained as follows: $w(x)=w_{\infty}(X, x)$. Substituting this solution in (15), we obtain

$$
P_{2}\left(\lambda_{\infty}(X, \cdot) ; x\right)=\omega_{\infty}(X, x) .
$$

Hence, $\left(\lambda_{\infty}(X, x), \omega_{\infty}(X, x)\right)$ expresses the equilibrium of full agglomeration at $X \in D$. Moreover, we see easily that (45) is a solution to the Cauchy problem for the logistic equation:

$$
\begin{aligned}
\left(\frac{d}{d t}\right) z(t) & =F(z(t)), \\
z(0) & =\lambda_{0}(X) .
\end{aligned}
$$

Hence, it follows from (57), (41), (42), and (43) that the economy converges to a full agglomeration equilibrium such that $\lambda=\lambda(t, X)$ increases with $t \geq 0$ more rapidly than the logistic curve defined by (59). However, from Corollary 2, we see that the economy does not coincide with such an equilibrium completely within a finite time interval. From (44) and (56) we see that $X$ gives the pure best reply and that the subpopulation associated with $X$ has the highest growth rate. Note that no condition is imposed on $\phi=\phi(x)$ in addition to (7) in Theorems 1 and 3.

\section{Proof of Main Results}

We define the constant $\beta$ as follows:

$$
\beta:=\theta \mathbf{H}_{+}^{1+\mu /(\sigma-1)}+R_{2}^{1 / \sigma} a,
$$

where

$$
\begin{aligned}
a & :=k\left(\frac{\mu}{(\sigma-1)}, \mathbf{H}_{-}, \mathbf{H}_{+}\right) b, \\
\mathbf{H}_{-} & :=\left(\frac{1}{R_{2}^{1-1 / \sigma}}\right) \exp (-\alpha), \\
\mathbf{H}_{+} & :=\frac{1}{R_{1}^{1-1 / \sigma}}, \\
b & :=\theta\left(1-\frac{1}{\sigma}\right) R_{1}^{-(2-1 / \sigma)}+\mathbf{H}_{+}, \\
k\left(s, r_{1}, r_{2}\right) & :=s r_{2}^{s-1} \text { if } s>1,0<r_{1} \leq r_{2}, \\
k\left(s, r_{1}, r_{2}\right) & :=r_{1}^{s-1} \text { if } 0<s \leq 1,0<r_{1} \leq r_{2},
\end{aligned}
$$

and $\theta$ denotes the positive constant defined in $[14,(7.20)-$ (7.23)].

Proof of (32). Applying [14, (5.11)], (5), and (6) to (62) and (63), we see that $0<\mathbf{H}_{-}<1<\mathbf{H}_{+}, b>1$. Applying these inequalities, (64), and (65) to (61), we obtain $a>1$. Applying this inequality and $[14,(5.11)]$ to (60), we obtain (32).

We make use of the following lemma in order to prove Theorems 1 and 3.
Lemma 4. If Conditions 1 and 2 hold, then the following statements (i) and (ii) hold:

(i) If (13) holds, then

$$
\begin{aligned}
m_{1} & \leq \omega(x) \leq m_{2}, \\
-\Delta m & \leq \mathbf{M}(\lambda(\cdot) ; x) \leq \Delta m,
\end{aligned}
$$

where

$$
\omega(x):=P_{2}(\lambda(\cdot) ; x) .
$$

(ii) If

$$
\lambda_{i}=\lambda_{i}(x) \in \Delta, \quad i=1,2,
$$

then

$$
\left\|\omega_{1}(\cdot)-\omega_{2}(\cdot)\right\| \leq \beta\left|\left\|\lambda_{1}(\cdot)-\lambda_{2}(\cdot)\right\|\right|,
$$

where

$$
\omega_{i}(x):=P_{2}\left(\lambda_{i}(\cdot) ; x\right), \quad i=1,2 .
$$

Proof. We can write (4) as follows:

$$
G_{\sigma}(\lambda(\cdot), w(\cdot) ; x)^{\sigma-1}=\frac{1}{H(\lambda(\cdot), W(\cdot) ; x)},
$$

where

$$
\begin{aligned}
& H(\lambda(\cdot), W(\cdot) ; x) \\
& :=\sum_{y \in D} \lambda(y)\left(\frac{1}{W(y)}\right)^{1-1 / \sigma} \exp (-(\sigma-1) c(x, y)), \\
& W(x):=w(x)^{\sigma} \\
& w(x):=P_{1}(\lambda(\cdot) ; x) .
\end{aligned}
$$

From $[14,(7.5)]$ we obtain

$$
\mathbf{H}_{-} \leq H(\lambda(\cdot), W(\cdot) ; x) \leq \mathbf{H}_{+} .
$$

Applying [14, (6.1)], (72), (74), and (75) to (15), we obtain (66). Applying (66) and (13) to (17), we obtain

$$
m_{1} \leq m(\lambda(\cdot), \omega(\cdot)) \leq m_{2} .
$$

From (66) and (76) we obtain (67). From [14, p. 491, first inequality] we see that

$$
\begin{aligned}
& \left\|H\left(\lambda_{1}(\cdot), W_{2}(\cdot) ; \cdot\right)-H\left(\lambda_{2}(\cdot), W_{2}(\cdot) ; \cdot\right)\right\| \\
& \quad \leq \mathbf{H}_{+}\left|\left\|\lambda_{1}(\cdot)-\lambda_{2}(\cdot)\right\|\right|,
\end{aligned}
$$

where

$$
\begin{aligned}
& W_{i}(x):=w_{i}(x)^{\sigma}, \\
& w_{i}(x):=P_{1}\left(\lambda_{i}(\cdot) ; x\right),
\end{aligned}
$$

$$
i=1,2 \text {. }
$$


Combine $[14,(7.3)]$ and $[14,(7.12)]$ with (69). Combining (77) and the inequality thereby obtained, we see that

$$
\begin{aligned}
& \left\|H\left(\lambda_{1}(\cdot), W_{1}(\cdot) ; \cdot\right)-H\left(\lambda_{2}(\cdot), W_{2}(\cdot) ; \cdot\right)\right\| \\
& \quad \leq b\left|\left\|\lambda_{1}(\cdot)-\lambda_{2}(\cdot)\right\|\right| .
\end{aligned}
$$

Making use of the mean value theorem, we see easily that if $g_{i}=g_{i}(x) \in L(D), i=1,2$, satisfy that $r_{1} \leq g_{i}(x) \leq r_{2}$ for each $x \in D, i=1,2$, where $0<r_{1} \leq r_{2}$, then

$$
\begin{aligned}
\left|g_{1}(x)^{s}-g_{2}(x)^{s}\right| \leq k\left(s, r_{1}, r_{2}\right)\left|g_{1}(x)-g_{2}(x)\right| & \\
s & >0 .
\end{aligned}
$$

Making use of (75), (79), and (80) with $g_{i}(x)=$ $H\left(\lambda_{i}(\cdot), W_{i}(\cdot) ; x\right), i=1,2, r_{1}=\mathbf{H}_{-}, r_{2}=\mathbf{H}_{+}, s=\mu /(\sigma-1)$, we obtain

$$
\begin{aligned}
& \| H\left(\lambda_{1}(\cdot), W_{1}(\cdot) ; \cdot\right)^{\mu /(\sigma-1)} \\
& \quad-H\left(\lambda_{2}(\cdot), W_{2}(\cdot) ; \cdot\right)^{\mu /(\sigma-1)}\left\|\leq a\left|\left\|\lambda_{1}(\cdot)-\lambda_{2}(\cdot)\right\|\right| .\right.
\end{aligned}
$$

It follows from (5), $[14,(5.11)]$, and (65) that $k\left(1 / \sigma, R_{1}, R_{2}\right)=$ $\mathbf{H}_{+}$. Apply this equality, $[14,(6.1)]$, and $[14,(7.3)]$ to (80) with $g_{i}(x)=W_{i}(x), r_{i}=R_{i}, i=1,2, s=1 / \sigma$. Performing calculations similar to those done in proving (81), we obtain

$$
\left\|w_{1}(\cdot)-w_{2}(\cdot)\right\| \leq \mathbf{H}_{+} \theta\left|\left\|\lambda_{1}(\cdot)-\lambda_{2}(\cdot)\right\|\right|
$$

Substitute (72) and $(\lambda(x), w(x), W(x), \omega(x))=\left(\lambda_{i}(x), w_{i}(x)\right.$, $\left.W_{i}(x), \omega_{i}(x)\right), i=1,2$, in (15). Subtract the equalities thereby obtained from each other. Applying [14, (6.1)], (75), (81), and (82) to the equality thereby obtained, we obtain (70).

Proof of Theorem 1. In [21, (11)-(13)] we consider the Cauchy problem for the replicator equation of spatially continuous evolutionary game whose payoffs are defined by the spatially continuous CP model defined in [13, (2.4)-(2.7)]. By analyzing this spatially continuous replicator equation in a Banach space defined in [21, p. 2] with the aid of [21, Lemma 8], we prove that if Conditions 1 and 2 hold (see [21, (5), (7), (8), (9), (22)]), then the Cauchy problem has a unique global solution in the Banach space, [21, Theorem 4]. We can regard Theorem 1 and Lemma 4 as a finite-dimensional version of [21, Theorem 4] and [21, (47), (49), Lemma 8]. Hence, applying Lemma 4 in place of [21, (47), (49), Lemma 8] to the Cauchy problem treated in Theorem 1 , and performing the same calculations as done in proving [21, Theorem 4], we obtain Theorem 1, (i), (25), (26), and (29). Apply (26) to the second term of the right-hand side of (52) with $\lambda(y)=$ $\lambda(t, y)$. Applying (52) with $\lambda(y)=\lambda_{0}(y)$ to the inequality obtained in this way, we obtain (27). Applying (25), (67), and (70) to the right-hand side of (19), we obtain (28) and (30).

In what follows we can make use of Theorem 1 and Corollary 2. Let $\lambda=\lambda(t, x)$ be a global solution proved in Theorem 1.
Lemma 5. If Conditions 1, 2, and 3 hold, then the following statements (i), (ii), and (iii) hold for each $Y \in D$ :

(i)

$$
\begin{aligned}
& \omega_{\infty}(Y, Y)=1, \\
& \omega_{\infty}(Y, x) \leq 1-\gamma \quad \text { for each } x \neq Y .
\end{aligned}
$$

(ii)

$\left|\left\|\lambda(t, \cdot)-\lambda_{\infty}(Y, \cdot)\right\|\right|=2(1-\lambda(t, Y))$

for each $t \geq 0$.

$$
\begin{aligned}
& \omega(t, Y) \geq 1-2 \beta(1-\lambda(t, Y)) \quad \text { for each } t \geq 0 \\
& \omega(t, x) \leq 1-\gamma+2 \beta(1-\lambda(t, Y)) \\
& \text { for each }(t, x) \in[0,+\infty) \times(D \backslash\{Y\}) .
\end{aligned}
$$

Proof. Applying (7) and (8) to (50), we obtain (83). It follows from (5), (6), (8), (10), and (34) that

$$
\exp (-\mu c(Y, x)) \leq\{\exp (-(\sigma-1) c(Y, x))\}^{1 / \sigma} .
$$

Applying this inequality to the right-hand side of (50) with $X=Y$, we see that

$$
\begin{aligned}
& \omega_{\infty}(Y, x) \leq\left\{\mu e^{-2(\sigma-1) c(Y, x)}\right. \\
& \left.+(1-\mu) \sum_{y \in D} \phi(y) e^{(\sigma-1)(c(Y, y)-c(Y, x)-c(x, y))}\right\}^{1 / \sigma} .
\end{aligned}
$$

Applying (7), (9), and (33) with $z=Y$ to the right-hand side of this equality, we see that

$$
\begin{array}{r}
\omega_{\infty}(Y, x) \leq\{1-\mu(1-\exp (-2(\sigma-1) c(Y, x)))\}^{1 / \sigma} \\
\text { for each } x \neq Y .
\end{array}
$$

Recalling definitions (38) and (37), we obtain (84).

It follows from (25) that

$$
0 \leq \lambda(t, x) \leq 1 \quad \text { for each } x \in D .
$$

Making use of (49) and (91), we can calculate the left-hand side of (85) as follows:

$$
\left\|\lambda(t, \cdot)-\lambda_{\infty}(Y, \cdot)\right\| \mid=1-\lambda(t, Y)+\sum_{y \in D, y \neq Y} \lambda(t, y) .
$$

Making use of (25) and (52), we replace the last term of the right-hand side of this equality with $1-\lambda(t, Y)$. Hence we obtain (85). Applying (58) with $X=Y$ to (70) with $\left(\lambda_{1}(x), \lambda_{2}(x)\right)=\left(\lambda(t, x), \lambda_{\infty}(Y, x)\right)$, we obtain

$$
\left\|\omega(t, \cdot)-\omega_{\infty}(Y, \cdot)\right\| \leq \beta\left|\left\|\lambda(t, \cdot)-\lambda_{\infty}(Y, \cdot)\right\|\right|
$$

for each $t \geq 0$. 
We rewrite this inequality as follows:

$$
\begin{aligned}
& \omega(t, Y) \geq \omega_{\infty}(Y, Y)-\beta\left|\left\|\lambda(t, \cdot)-\lambda_{\infty}(Y, \cdot)\right\|\right| \\
& \text { for each } t \geq 0 \text {, } \\
& \omega(t, x) \leq \omega_{\infty}(Y, x)+\beta\left|\left\|\lambda(t, \cdot)-\lambda_{\infty}(Y, \cdot)\right\|\right| \\
& \text { for each }(t, x) \in[0,+\infty) \times(D \backslash\{Y\}) \text {. }
\end{aligned}
$$

Applying (83), (84), and (85) to the right-hand sides of these inequalities, we obtain (86) and (87).

Lemma 6. Assume that Conditions 1, 2, and 3 hold and that $X \in D$ satisfies (35). If there exists $\tau \geq 0$ such that

$$
\lambda(\tau, X) \geq \lambda_{0}(X)
$$

then

$$
\begin{aligned}
& \omega(\tau, X) \geq 1-\frac{\kappa \gamma}{2}, \\
& \omega(\tau, y) \leq 1-\gamma+\frac{\kappa \gamma}{2}
\end{aligned}
$$

$$
\text { for each } y \neq X \text {, }
$$

$$
\mathbf{M}(\lambda(\tau, \cdot) ; X) \lambda(\tau, X) \geq F(\lambda(\tau, X)) .
$$

Proof. Substitute (36) in (47). Apply (95) and the equality thereby obtained to the right-hand sides of (86) and (87) with $(t, Y)=(\tau, X)$, and we obtain (96) and (97). Decompose the right-hand side of (17) as follows:

$$
\begin{aligned}
m(\lambda(t, \cdot), \omega(t, \cdot))= & \omega(t, X) \lambda(t, X) \\
& +\sum_{y \in D, y \neq X} \lambda(t, y) \omega(t, y) .
\end{aligned}
$$

Substituting this equality in $(20)$ when $(t, x)=(\tau, X)$, we see that

$$
\begin{aligned}
M & (\lambda(\tau, \cdot), \omega(\tau, \cdot) ; X) \\
= & \omega(\tau, X)(1-\lambda(\tau, X)) \\
& +\sum_{y \in D, y \neq X} \lambda(\tau, y)(-\omega(\tau, y)) .
\end{aligned}
$$

Multiply both sides of (97) by -1 . Making use of the inequality obtained in this way, we replace $-\omega(\tau, y)$ with $-(1-\gamma+\kappa \gamma / 2)$ in the last term of the right-hand side of (100). Moreover, with the aid of (52), we replace $\sum_{y \in D, y \neq X} \lambda(\tau, y)$ with $1-\lambda(\tau, X)$ in the inequality thereby obtained. Apply (91) and (96) to the first term of the right-hand side of the inequality obtained in this way. Recalling (56), (46), and (48), we obtain (98).

Proof of Theorem 3. It follows from (55), (35), and Corollary 2 that $0<\lambda(t, X)<1$ for each $t \geq 0$. Applying this inequality and (56) to (48), we see that

$$
F(\lambda(t, X))>0 \text { for each } t \geq 0 .
$$

Applying Theorem 1, (i), and (70) to the right-hand side of (19), we see that

$$
\left(\frac{d}{d t}\right) \lambda(t, x) \text { is continuous with respect to } t \geq 0
$$

$$
\text { for each } x \in D \text {. }
$$

Note that (95) holds when $\tau=0$. Applying (101), (102), and (98) with $\tau=0$ to (19) with $(t, x)=(0+0, X)$, we see that $\lim _{t \rightarrow 0+0}(d / d t) \lambda(t, X)>0$. Combining this inequality and (102), we see that

$$
\begin{aligned}
S:= & \sup \left\{s>0 ;\left(\frac{d}{d t}\right) \lambda(t, X)>0 \text { for each } t\right. \\
& \in[0, s]\}>0 .
\end{aligned}
$$

Assume that

$$
S<+\infty \text {. }
$$

It follows from the inequality contained in the definition of (103) that (95) holds for each $\tau \in[0, S]$. Making use of Lemma 6, we obtain (98) for each $\tau \in[0, S]$. Combining this result and (19), we obtain (42) for each $t \in[0, S]$. From this result and (101), we deduce that the inequality contained in the definition of (103) holds at $t=S$. This result, (102), (103), and (104) lead us to a contradiction. Hence, $S=+\infty$. Making use of this result, (19), and Lemma 6, we obtain (42) for each $t \geq 0$. Solving this differential inequality with (22), we obtain (39). From (25) we see that

$$
0 \leq \lambda(t, y) \leq 1-\lambda(t, X) \text { for each } y \neq X .
$$

Making use of (39) and (105), we obtain (40). Applying (39) to the right-hand side of (85) with $Y=X$, we obtain (41). Combining (41) and (93), we obtain (43). Combining (96) and (97) for each $\tau \geq 0$, we obtain (44).

\section{Competing Interests}

The authors declare that there are no competing interests regarding the publication of this paper.

\section{Acknowledgments}

This work is supported by Grant-in-Aid for Scientific Research (15K05005; 26330045), Ministry of Education, Culture, Sports, Science and Technology of Japan.

\section{References}

[1] M. Burger, L. Caffarelli, and P. A. Markowich, "Partial differential equation models in the socio-economic sciences," Philosophical Transactions of the Royal Society A: Mathematical, Physical and Engineering Sciences, vol. 372, no. 2028, Article ID 20130406, 2014.

[2] S. B. S. D. Castro, J. Correia-da-Silva, and P. Mossay, "The core-periphery model with three regions and more," Papers in Regional Science, vol. 91, no. 2, pp. 401-418, 2012. 
[3] N. G. Pavlidis, M. N. Vrahatis, and P. Mossay, "Existence and computation of short-run equilibria in economic geography," Applied Mathematics and Computation, vol. 184, no. 1, pp. 93103, 2007.

[4] M. Fujita, P. Krugman, and A. J. Venables, The Spatial Economy, The MIT Press, Cambridge, Mass, USA, 1999.

[5] M. Fujita and J.-F. Thisse, Economics of Agglomeration, Cities, Industrial Location, and Regional Growth, Cambridge University Press, Cambridge, UK, 2002.

[6] M. Fujita, "The evolution of spatial economics: from Thünen to the new economic geography," The Japanese Economic Review, vol. 61, no. 1, pp. 1-32, 2010.

[7] K. Ikeda, T. Akamatsu, and T. Kono, "Spatial period-doubling agglomeration of a core-periphery model with a system of cities," Journal of Economic Dynamics and Control, vol. 36, no. 5, pp. 754-778, 2012.

[8] P. Krugman, The Self-Organizing Economy, Blackwell, 1996.

[9] P. Krugman, "Increasing returns and economic geography," The Journal of Political Economy, vol. 99, no. 3, pp. 483-499, 1991.

[10] P. Mossay, "Increasing returns and heterogeneity in a spatial economy," Regional Science and Urban Economics, vol. 33, no. 4, pp. 419-444, 2003.

[11] P. Mossay, "The core-periphery model: a note on the existence and uniqueness of short-run equilibrium," Journal of Urban Economics, vol. 59, no. 3, pp. 389-393, 2006.

[12] P. Mossay and T. Tabuchi, "Preferential trade agreements harm third countries," The Economic Journal, vol. 125, no. 589, pp. 1964-1985, 2015.

[13] M. Tabata, N. Eshima, Y. Sakai, and I. Takagi, "An extension of Krugman's core-periphery model to the case of a continuous domain: existence and uniqueness of solutions of a system of nonlinear integral equations in spatial economics," Nonlinear Analysis: Real World Applications, vol. 14, no. 6, pp. 2116-2132, 2013.

[14] M. Tabata, N. Eshima, Y. Kiyonari, and I. Takagi, “The existence and uniqueness of short-run equilibrium of the Dixit-StiglitzKrugman model in an urban-rural setting," IMA Journal of Applied Mathematics, vol. 80, no. 2, pp. 474-493, 2015.

[15] M. Tabata, N. Eshima, and Y. Sakai, "Existence, uniqueness, and computation of short-run and long-run equilibria of the Dixit-Stiglitz-Krugman model in an urban setting," Applied Mathematics and Computation, vol. 234, pp. 339-355, 2014.

[16] R. Boucekkine, C. Camacho, and G. Fabbri, "Spatial dynamics and convergence: the spatial AK model," Journal of Economic Theory, vol. 148, no. 6, pp. 2719-2736, 2013.

[17] C. Camacho, "Migration modelling in the new economic geography," Mathematical Social Sciences, vol. 66, no. 3, pp. 233244, 2013.

[18] M. Tabata and N. Eshima, "A population explosion in an evolutionary game in spatial economics: blow up radial solutions to the initial value problem for the replicator equation whose growth rate is determined by the continuous Dixit-StiglitzKrugman model in an urban setting," Nonlinear Analysis: Real World Applications, vol. 23, pp. 26-46, 2015.

[19] M. Tabata, N. Eshima, and Y. Sakai, "Existence and computation of solutions to the initial value problem for the replicator equation of evolutionary game defined by the Dixit-StiglitzKrugman model in an urban setting: concentration of workers motivated by disparity in real wages," Applied Mathematics and Computation, vol. 254, pp. 419-451, 2015.
[20] J. W. Weibull, Evolutionary Game Theory, MIT Press, Boston, Mass, USA, 1997.

[21] M. Tabata and N. Eshima, "The existence and uniqueness of global solutions to the initial value problem for the system of nonlinear integropartial differential equations in spatial economics: the dynamic continuous Dixit-Stiglitz-Krugman model in an urban-rural setting," Abstract and Applied Analysis, vol. 2015, Article ID 760136, 12 pages, 2015. 


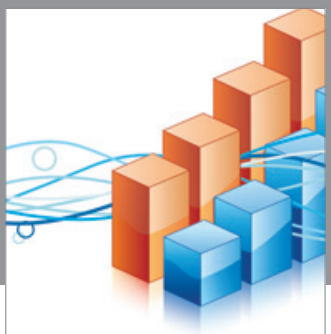

Advances in

Operations Research

vatem alat4

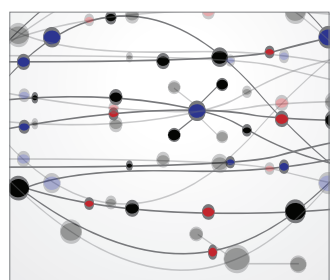

\section{The Scientific} World Journal
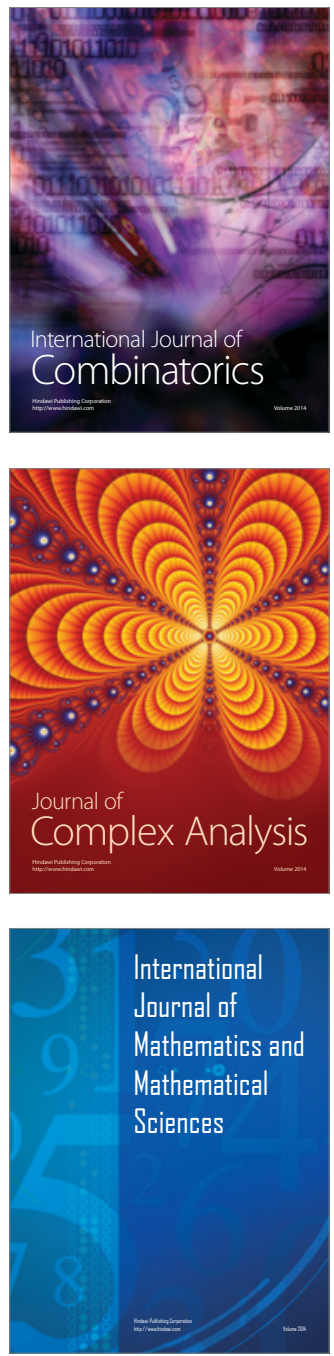
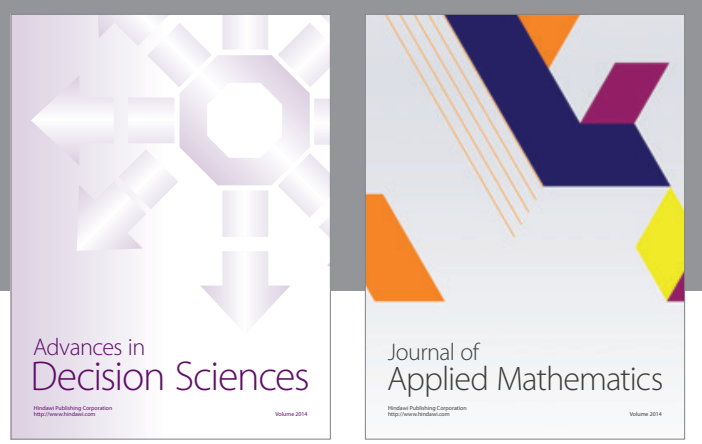

Algebra

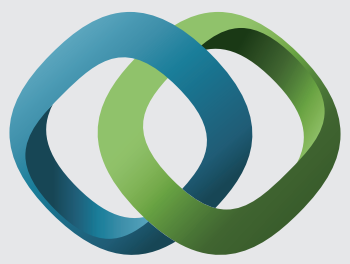

\section{Hindawi}

Submit your manuscripts at

http://www.hindawi.com
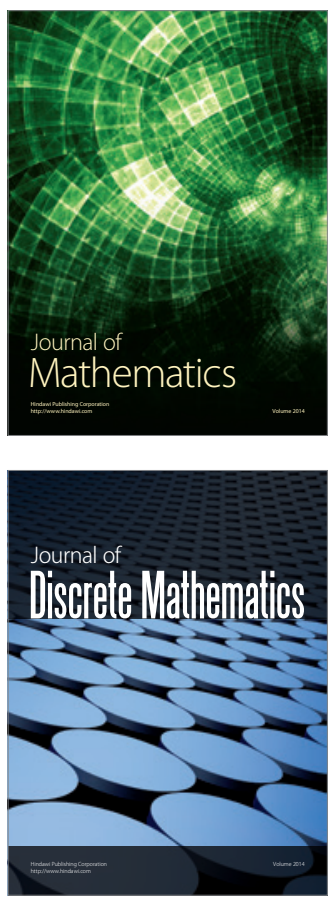

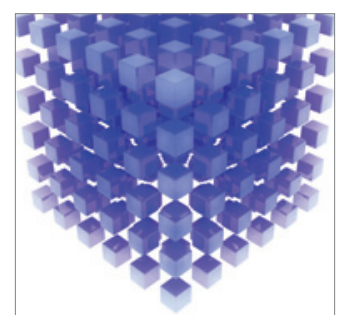

Mathematical Problems in Engineering
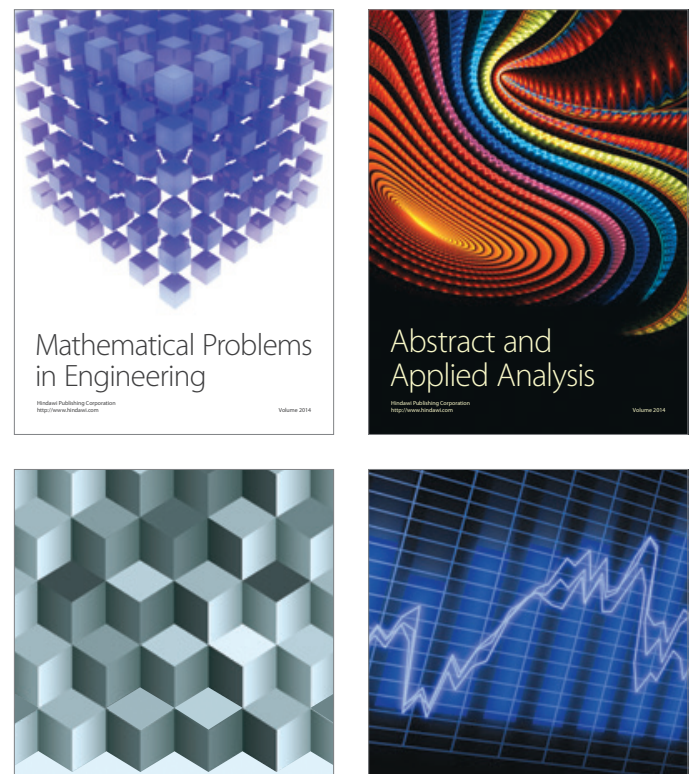

Journal of

Function Spaces

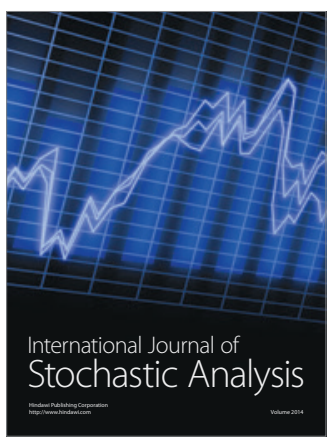

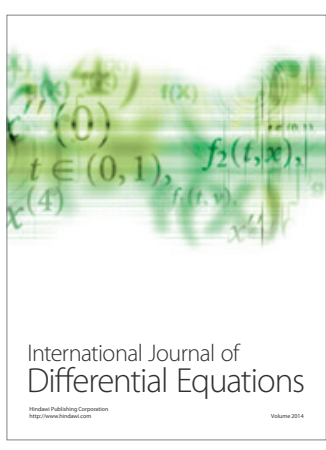
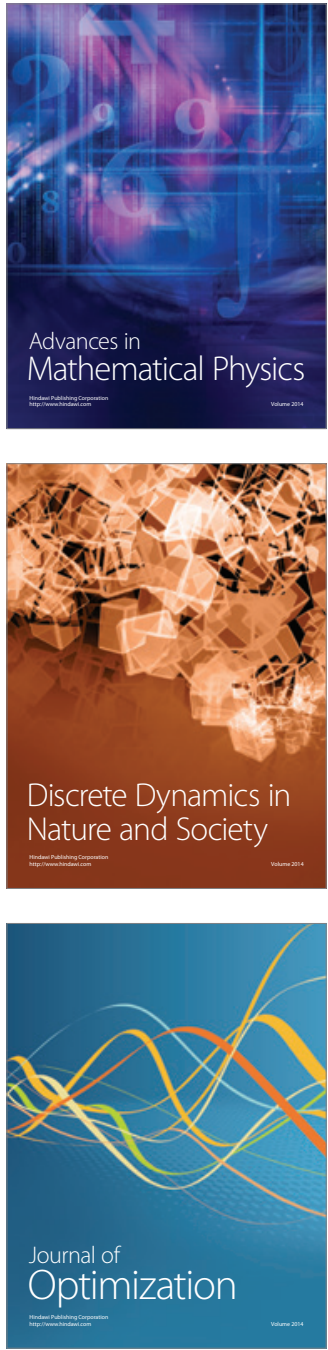